\title{
Programmed death ligand-1, tumor infiltrating lymphocytes and HLA expression in Chinese extrahepatic cholangiocarcinoma patients: Possible immunotherapy implications
}

\author{
Fei Yu ${ }^{1}$, Lei Gong ${ }^{2}$, Zheng Mo ${ }^{3}$, Wenran Wang ${ }^{1,2}$, Meilong Wu ${ }^{1,2}$, Jianghui Yang ${ }^{4}$, \\ Qiongqiong Zhang ${ }^{1}$, Li Li ${ }^{4}$, Jingjing $\mathrm{Yao}^{4}$, Jiahong Dong ${ }^{1, *}$ \\ ${ }^{1}$ School of Clinical Medicine, Tsinghua University, Beijing, China; \\ ${ }^{2}$ Department of Hepatopancreatobiliary Surgery, Beijing Tsinghua Changgung Hospital, School of Clinical Medicine, Tsinghua \\ University, Beijing, China; \\ ${ }^{3}$ Department of Hematology and Oncology, Beijing Tsinghua Changgung Hospital, School of Clinical Medicine, Tsinghua \\ University, Beijing, China; \\ ${ }^{4}$ Department of Pathology, Beijing Tsinghua Changgung Hospital, School of Clinical Medicine, Tsinghua University, Beijing, \\ China.
}

Summary Immunotherapy might be an effective treatment in extrahepatic cholangiocarcinoma (eCCA), a tumor with extremely limited therapeutic options. Our study is to characterize the programmed death ligand-1 (PD-L1) protein expression and cancer microenvironment profiles in surgically resected eCCA samples. PD-L1 positivity was observed on tumor cells $(32.3 \%)$ as well as on tumor-associated macrophages $(74.2 \%)$. PD-L1 expression by eCCA correlated significantly with immune parameters such as intra-tumoral CD3+ tumor infiltrating lymphocytes (TILs) density $(P=0.002)$, intra-tumoral CD8+ TILs density $(P<$ $0.001)$, and the expression pattern of human leukocyte antigen (HLA) class $I(P<0.001)$. Immunofluorescence showed that PD-L1 positive tumor cells were adjacent to PD-1 positive cells and the stroma covered with interferon- $\gamma$. Correlation with clinicopathological parameters and survival analyses revealed that PD-L1 positivity in eCCA was related to the absence of venous invasion $(P=0.030)$, improved overall survival $(P=0.020)$ and progressionfree survival $(P=\mathbf{0 . 0 1 1})$. HLA class I molecules defect, which is an important mechanism of immune evasion, was frequently observed in eCCA $(50.0 \%)$ and was associated with a decreased number of intra-tumoral CD8+ TIL density $(P=\mathbf{0 . 0 2 8})$. Additionally, the presence of unusually high numbers of tumor-associated macrophages (TAMs) subsets M2 in most of eCCA (74.2\%) was noted. Our study indicated that PD-L1 expression in association with intra-tumoral TILs infiltration and HLA class I expression in $32.3 \%$ of the eCCA reflects an active immune microenvironment potentially responsive to $\mathrm{PD}-1 / \mathrm{PD}-\mathrm{L} 1$ inhibitors. In addition, the combination of macrophage-targeting agents may provide therapeutic synergy for future immunotherapy.

Keywords: Extrahepatic cholangiocarcinoma, PD-L1, TILs, HLA class I molecules, TAMs

\section{Introduction}

Cholangiocarcinoma (CCA) is a rare cancer biliary

Released online in J-STAGE as advance publication February $15,2019$.

*Address correspondence to:

Dr. Jiahong Dong, School of Clinical Medicine, Tsinghua University, No.1, Tsinghua Yuan, Haidian District, Beijing, 100084, China.

E-mail: djhtsinghua@163.com tract malignancy which accounts for less than $3 \%$ of all gastrointestinal cancers diagnosed worldwide (1). The incidence of CAA is highest in Thailand, China, and other Asian populations, but is lowest in the western world, presumably reflecting differences in the exposure of genetic and other risk factors (2). Anatomically, CCA can be divided into intrahepatic or extrahepatic subtypes choosing second-order bile ducts as the point of separation (3). The majority of CCA are extrahepatic cholangiocarcinoma (eCCA), whereas 
intrahepatic cholangiocarcinoma (iCC) comprise less than $10 \%$ of cases (4). eCCA can be classified further into perihilar (pCCA) and distal tumors (eCCA), separated by the cystic duct (5).

Despite advances in development of early detection methodologies, CCA continues to be a challenging malignancy with extremely limited treatment options. A minority of patients at a sufficiently early stage have a chance of curative surgical resection, especially those with localized disease without vascular and/or parenchymal involvement (6). Neoadjuvant chemoradiotherapy followed by liver transplantation offers another therapeutic option that can achieve complete extirpation of locally advanced pCCA in judiciously selected patients (7). For patients beyond the stringent inclusion criteria, no other curative treatment has proved successful (8). Locally advanced or metastatic CCA can be treated with chemotherapeutic regimens but is of limited effectiveness, unlike the improvement in survival achieved in colorectal and gastric cancers (9). Recent genomic sequencing technologies have facilitated the identification of therapeutic targets in various solid tumors, implicating a promising strategy for CCA (10). However, success rate from bench to bedside has so far remained significantly low due to lack of objective clinical response (11).

Monoclonal antibodies directed at the programmed cell death protein 1 (PD-1) immune checkpoint and one of its ligands, PD-L1, has demonstrated impressive antitumor responses (12). By activating T cell-mediated immunity, some meaningful anti-tumor responses were observed in late-stage melanoma - a type of cancer largely resistant to standard chemotherapy (13). Subsequently, the clinical value of the PD-1/PD-L1 axis has been extended in non-small cell lung cancer and other solid tumors (14). However, to date there is scarce data regarding the expression of PD-L1 and its role in tumor microenvironment of eCCA (15). Recently, Dirk Walter et al reported a relatively low frequency of PD-L1 positivity $(8 / 69,11.6 \%)$ in eCCA arising in the European population (16). However, this positive rate cannot be generalized to other regions due to ethnic disparities of molecular patterns among eCCA patients (17). Therefore, further analysis of PD-L1 expression in eCCA in populations with different ethnic backgrounds was necessary, as it might personalize the treatment for this ethnic -heterogeneous disease.

The overexpression of PD-L1 in a tumor site may predict the response to PD-1/PD-L1 antibodies $(18,19)$. However, the determinants of reliable response or primary resistance to immune checkpoint inhibition are clearly multi-faceted (20). For example, Tumor-infiltrating lymphocytes (TILs) are the main executioners of antitumor immunity, but reduced expression of major histocompatibility complex (MHC; also known as human leukocyte antigen (HLA) in humans) can render the malignant cells "invisible" to the immune system (21). TILs as well as HLA antigens have been reported to be predictive markers for PD-1/ PD-L1 checkpoint blockades in many cancers, but no study has, to our knowledge, evaluated these important prerequisites in a clinicopathologic study of eCCA, so far $(16,22)$.

This study was performed to characterize the expression of PD-L1 in a Chinese cohort of eCCA patients, and to correlate the results with the clinicopathological findings including the outcome. Furthermore, to support a rationale regarding the application of PD-1/PD-L1 blockades, we assessed some important immune modulators in the tumor microenvironment such as TILs, tumor-associated macrophages (TAMs) and HLA-I expression.

\section{Materials and Methods}

\subsection{Patient material}

The current study evaluated formalin-fixed paraffinembedded (FFPE) tumor sections from eCCA patients who underwent curative-intent surgery from January 2015 to September 2017, in Beijing Tsinghua Changgung Hospital affiliated with Tsinghua University, one of the leading Chinese medical centers for hepatobiliary surgery. None of the patients had received any neoadjuvant treatment followed by curative resection. Pathologic diagnoses were confirmed by a pathologist (H. F. Yin) according to the 7th American Joint Committee on Cancer (AJCC) and the histologic types were determined by the WHO classification. Patients with other primary tumors of hepatobiliary system such as Gallbladder and/or ampullary carcinoma, were excluded from the current study.

For the patients enrolled, clinicopathologic information available included demographic information (age and sex), AJCC stage, grading (G), presence of venous invasion $(\mathrm{V})$, presence of perineural invasion $(\mathrm{P})$, and tumor infiltration of resection margins (R). Progression-free survival (PFS) is defined by the interval from surgery to recurrence deadline date of the study (October 2018). Overall survival (OFS) is defined as the duration from surgery to date of death or deadline date of the study. Informed consent for the use of samples was obtained individually from each patient. This retrospective study was approved with the agreement of ethics committee of the Beijing Tsinghua Changgung Hospital affiliated to Tsinghua University.

Tumor samples from a cohort of 62 surgically resected eCCA (45 pCCA and $17 \mathrm{dCCA}$ ) were analyzed. The group included 41 males $(66.1 \%)$ and 21 females $(33.9 \%)$, aged between 22 and 81 years (mean age, 60.8 years). The perioperative mortality was $6.5 \%$ (4 patients) and thus those patients were not included 
in further survival analysis. Kaplan-Meier survival analysis according to clinicopathological characteristics indicated that our cases are representative of an average eCCA population and thus provide a better baseline for the retrospective analysis (Figure $\mathrm{S} 1, h t t p: / / w w w$. biosciencetrends.com/action/getSupplementalData. php?ID=37).

\subsection{Immunohistochemistry and scoring}

After review of all surgically resected specimens stained with hematoxylin and eosin (H\&E), a representative slide with a sufficient eCCA fraction was chosen for research. 4- $\mu$ m paraffin embedded tissues were deparaffinized, cleared and rehydrated through decreasing concentration of ethanol (100\%, $95 \%, 85 \%$, and $75 \%$ ). Heat-induced epitope retrieval was performed in a solution of EDTA, pH 8.0. Monoclonal primary antibodies against (PD-L1, 1/200, E1L3N, Cell Signaling Technology, Danvers, MA, USA; CD3, 1/150, ab16669, Abcam, Cambridge, UK; CD4, 1/400, ab133616, Abcam, Cambridge, UK; CD8, 1/200, ab17147, Abcam, Cambridge, UK; CD163, 1/500, EPR19518, Abcam, Cambridge, UK; HLA Class one, 1/300, EMR8-5, Abcam, Cambridge, UK) were incubated overnight at $4^{\circ} \mathrm{C}$. Detection of immunolabeling was performed by incubation with biotinylated secondary antibodies (SignalStain ${ }^{\circledR}$ Boost IHC Detection Reagent; Cell Signaling Technology, Danvers, MA, USA). To verify antibody specificity, positive controls consisted of human placental tissue for PD-L1 and human tonsil tissues for the marker of TILs. Endothelial cells or T cells were included to serve as a positive internal control for HLA class I antibody. The stained slides were scanned with a high throughput digital slide scanner (Pannoramic 250 Flash III and Viewer, 3DHISTECH) to obtain a whole slide image. All sections were examined and scored independently by two pathologists (J.J.Y. and L.L) blinded to the clinical data. When coming to a point of discrepancies, re-evaluation and discussion were conducted to reach a consensus opinion.

The expression of PD-L1 was assessed on tumor cells, lymphocytes as well as on macrophages. Cells expressing PD-L1 on membranes were regarded as positive, while cytoplasmic staining only was termed as negative. For tumor cells positivity, the intensity ( 0 , no staining; 1 , weak; 2 , moderate; 3 , intense) and the percentage of membranous positivity $(0-100 \%)$ were considered collectively. Total immunostaining score (TIS) was calculated by multiplying the percentage of the positive cells by the staining intensity. TIS score $(0-300)$, with a score $\geq 3$ being defined as PD-L1 positivity according to recent studies with the same antibody $(16,23)$.

Instead of measuring TILs dichotomically, we quantified the density of TILs by averaging the number of positive cells (CD3, CD4 and CD8) within at least five HPF areas (Perimeter: $1960.0 \mu \mathrm{m}$, Area: 0.305 $\mathrm{mm}^{2}$ ). To reduce heterogeneity, both inside the tumor and surrounding the cancer nest were scored separately when possible. TAMs were classified into low or high groups based upon the cut-off value of $>20 \% \mathrm{CD} 163+$ cells as previously described (24).

HLA class I expression was divided into three criteria for membrane expression as previously described: negative $(<25 \%$ HLA positive tumor cells), heterogeneous (between $25 \%$ and $75 \%$ HLA positive tumor cells) or positive ( $>75 \%$ HLA positive tumor cells) $(25,26)$. Positive or heterogeneous HLA class I expression were assigned to the "high HLA-I expression" group while negative expression was defined as the "low HLA-I expression" group.

\subsection{Immunofluorescent staining and scoring}

After deparaffinization, rehydration and heat-induced antigen retrieval, tissue samples were incubated overnight with the primary anti-human PD-L1 (Rabbit mAb, 1/200, E1L3N, Cell Signaling Technology, Danvers, MA, USA) combined with anti-human PD-1 (Mouse mAb, 1/100, ab52587, Abcam, Cambridge, $\mathrm{UK}$ ), or anti-human PD-L1 (Mouse mAb, 1/100, EMR8-5, Abcam, Cambridge, UK) combined with anti-human interferon $\gamma$ (IFN- $\gamma$ ) (Rabbit mAb, 1/200, ab9657, Abcam, Cambridge, UK). Slides were then incubated with secondary antibodies: goat anti-rabbitFITC (ab6717, Abcam, Cambridge, UK) and goat antimouse-TRITC (ab6786, Abcam, Cambridge, UK). Nuclei were stained with 4,6-diamidino-2-phenylindole (\#4083, Cell Signaling Technology, Danvers, MA, USA). Fluorescent images were analyzed with a fluorescence microscope (BX53, Olympus).

In humans, macrophages polarize into two extremes from the classically activated M1 macrophages (CD14+ CD163-) to the alternatively activated M2 macrophages (CD14+ CD163+) (27). To define macrophage polarization, CD14 (Mouse mAb, 1/300, ab182032, Abcam, Cambridge, UK) was used as macrophage surface marker, and polarized macrophage was identified by expression of the CD163 (Rabbit mAb, 1/500, ab182422, Abcam, Cambridge, UK): CD14+CD163+ cells indicated the immunosuppressive M2 subset while CD14+CD163- cells were considered as the immunostimulatory M1 subset. The numbers of CD14 positive cells and CD163 positive cells were counted respectively in five representative visual fields with highpower fields $(\mathrm{HPF} \times 400)$.

\subsection{Statistical analysis}

The associations of PD-L1 expression with each clinicopathological characteristic were analyzed with the $\chi^{2}$ test or Fisher's exact test, as appropriate. Mann- 

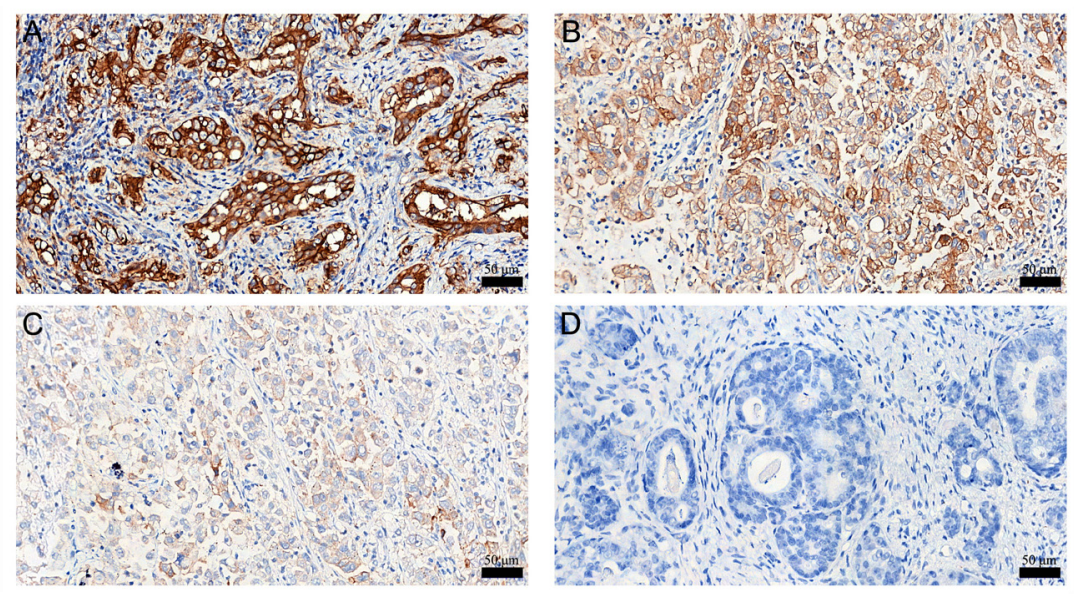

Figure 1. Representative immunohistochemical images for PD-L1 in eCCA with strong (A), moderate (B), weak (C) and no (D) PD-L1 expression. In these tissues, PD-L1 showed membrane-accentuated expression, also often accompanied by cytoplasmic expression. Cells with PD-L1 expression on the membrane were assessed as PD-L1 positive, whereas only cytoplasmic PD-L1 expression were assessed as PD-L1 negative.

DAPI

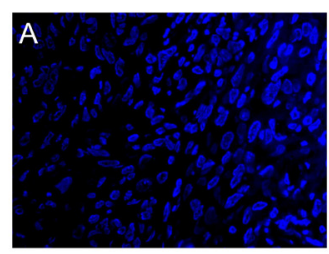

DAPI

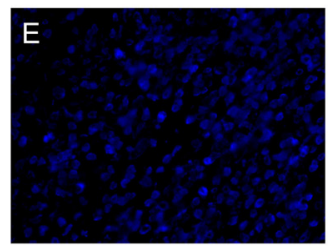

CK7

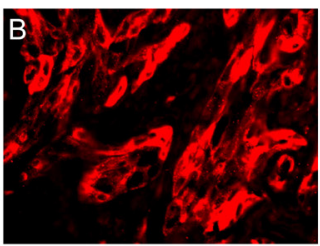

CD163

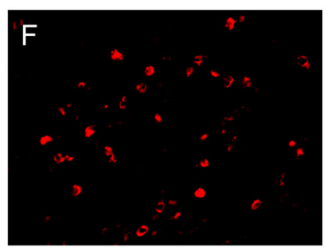

PD-L1

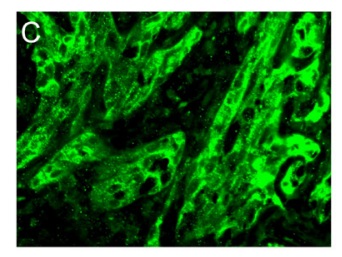

PD-L1

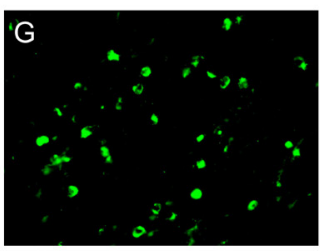

Merged

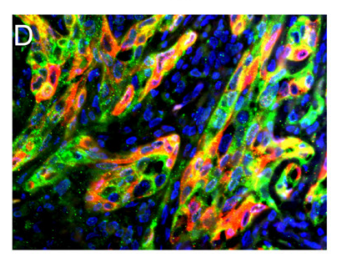

Merged

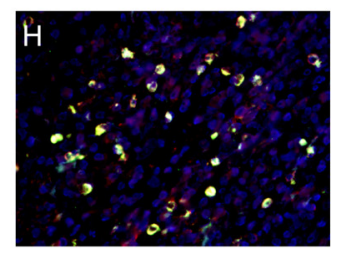

Figure 2. Identification of PD-L1-positive tumor cells (A-D) and tumor-infiltrating macrophages (TAMs) (E-H) in eCCA. Representative double immunofluorescence staining shows monochromatic PD-L1 (in green), CK7(in red), CD163(in red) images, and colocalized PD-L1/CK7, PD-L1/CD163, and DAPI (4',6-diamidino-2-phenylindole) (in blue).

Whitney $U$-test was used to detect differences in density of the TILs between groups of patients. KaplanMeier survival curves were generated and log-rank test was used for assessing PD-L1 in relation to overall and progression-free survival. $P$-values were two-sided, and $P$-values of $<0.05$ were considered statistically significant. The statistical analyses were performed using SPSS version 20.0 software (Chicago, IL).

\section{Results}

3.1.PD-L1 expression in eCCA and its association with eCCA clinicopathologic features

PD-L1 positive cells were identified in 46 patients analyzed $(46 / 62,74.2 \%)$; however, in only 20 patients (20/62, 32.3\%) PD-L1 positivity was expressed on tumor cells (Figure 1A-D). In these 20 PD-L1 positive eCCA, PD-L1 was co-expressed by tumor cells as well as a few macrophages. For the remaining 26 eCCA, PDL1 positivity was merely expressed by macrophages. Expression of PD-L1 by both tumor cells and macrophages was confirmed by PD-L1 and CK7, and PD-L1 and CD163 double-immunofluorescence staining (Figure 2A-D, E-H). Tumor cell staining for PD-L1 occurred in two patterns: focal $(17 / 20,85.0 \%)$ or diffuse (3/20, 15.0\%) (Figure S2, http://www.biosciencetrends. com/action/getSupplementalData.php? ID=37). On investigation of clinicopathological features, significant correlations between tumoral PD-L1 expression and absence of venous invasion was observed $(\mathrm{P}=0.030)$ (Table 1).

To evaluate the correlation between PD-L1 in eCCA specimens $(n=58)$ and the prognosis of eCCA patients, 
Table 1. Correlation between PD-L1 expression and variable clinicopathologic parameters in 62 eCCA

\begin{tabular}{|c|c|c|c|c|c|c|c|}
\hline Variable & Patients (total) & $\%$ & PD-L1 positive tumor & $\%$ & PD-L1 negative tumor & $\%$ & $P$-value \\
\hline \multicolumn{8}{|l|}{ Age (years) } \\
\hline$\geq 60$ & 37 & 59.7 & 7 & 63.6 & 30 & 58.8 & 0.971 \\
\hline$<60$ & 25 & 40.3 & 4 & 36.4 & 21 & 59.7 & \\
\hline \multicolumn{8}{|l|}{ Gender } \\
\hline Male & 41 & 66.1 & 13 & 65.0 & 28 & 66.7 & 0.897 \\
\hline Female & 21 & 33.9 & 7 & 35.0 & 14 & 33.3 & \\
\hline \multicolumn{8}{|c|}{ Localization } \\
\hline pCCA & 45 & 72.6 & 13 & 65.0 & 32 & 76.2 & 0.356 \\
\hline $\mathrm{dCCA}$ & 17 & 27.4 & 7 & 35.0 & 10 & 23.8 & \\
\hline \multicolumn{8}{|l|}{ Grading } \\
\hline G1-G2 & 46 & 74.2 & 9 & 81.8 & 33 & 78.6 & 0.353 \\
\hline G3 & 16 & 25.8 & 2 & 18.2 & 9 & 21.4 & \\
\hline \multicolumn{8}{|c|}{ Tumour size (mm) } \\
\hline$\geq 30$ & 18 & 29.0 & 8 & 40.0 & 10 & 23.8 & 0.236 \\
\hline$<30$ & 44 & 71.0 & 12 & 60.0 & 32 & 76.2 & \\
\hline \multicolumn{8}{|c|}{ Venous invasion } \\
\hline V0 & 31 & 50.0 & 14 & 70.0 & 25 & 59.5 & $0.030 *$ \\
\hline V1 & 31 & 50.0 & 6 & 30.0 & 17 & 40.5 & \\
\hline \multicolumn{8}{|c|}{ Perineural invasion } \\
\hline PNO & 11 & 17.7 & 5 & 25.0 & 6 & 14.3 & 0.311 \\
\hline PN1 & 51 & 82.3 & 15 & 75.0 & 36 & 85.7 & \\
\hline \multicolumn{8}{|c|}{ Lymph node metastasis } \\
\hline N0 & 34 & 54.8 & 12 & 60.0 & 22 & 52.4 & 0.598 \\
\hline N1 & 28 & 45.2 & 8 & 40.0 & 20 & 47.6 & \\
\hline \multicolumn{8}{|c|}{ Resection margin } \\
\hline R0 & 48 & 77.4 & 18 & 81.8 & 30 & 76.5 & 0.192 \\
\hline $\mathrm{R} 1$ & 14 & 22.6 & 2 & 18.2 & 12 & 23.5 & \\
\hline \multicolumn{8}{|c|}{ AJCC classification } \\
\hline I-II & 23 & 37.1 & 10 & 50.0 & 13 & 31.0 & 0.169 \\
\hline III- IV & 39 & 62.9 & 10 & 50.0 & 29 & 69.0 & \\
\hline \multicolumn{8}{|c|}{ CD163 infiltrate } \\
\hline High & 42 & 67.7 & 18 & 90.0 & 33 & 78.6 & 0.478 \\
\hline Low & 20 & 32.3 & 2 & 10.0 & 9 & 21.4 & \\
\hline \multicolumn{8}{|c|}{ HLA expression } \\
\hline High & 44 & 71.0 & 20 & 100.0 & 0 & 0.0 & $<0.001 *$ \\
\hline Low & 18 & 29.0 & 24 & 57.1 & 18 & 42.9 & \\
\hline
\end{tabular}

A

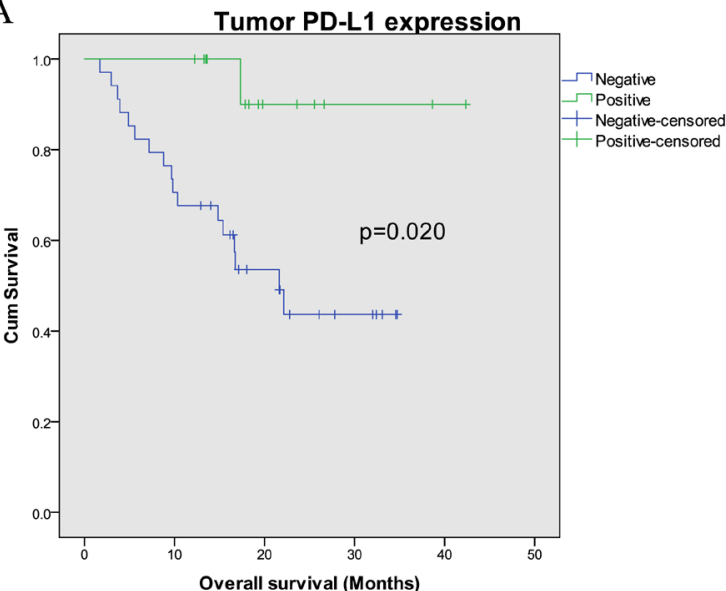

$\mathrm{B}$

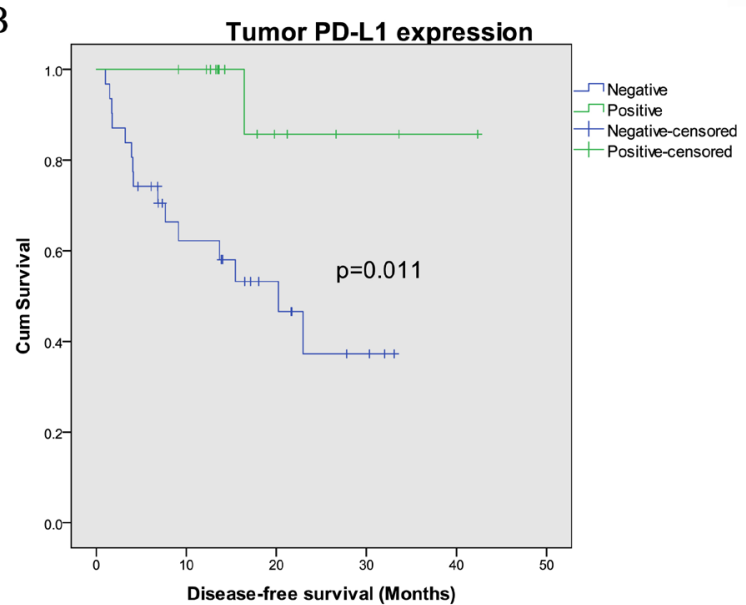

Figure 3. Association of PD-L1 expression and post-treatment outcome (A and B) of eCCA. PD-L1 positivity in tumor cells is associated with better overall and progression-free survival. P value obtained by log-rank test.

Kaplan-Meier survival curves were generated. Patients with PD-L1 expression at the tumor site showed better overall and progression-free survival (log rank test; $P=$ 0.020 and 0.011 respectively) compared to those with negative PD-L1 expression (Figure 3A-B).
3.2. Correlation between TIL subtype densities and PDL1 expression in eCCA

To reveal the correlation between PD-L1 expression and immune infiltration in eCCA, staining of TIL 
A
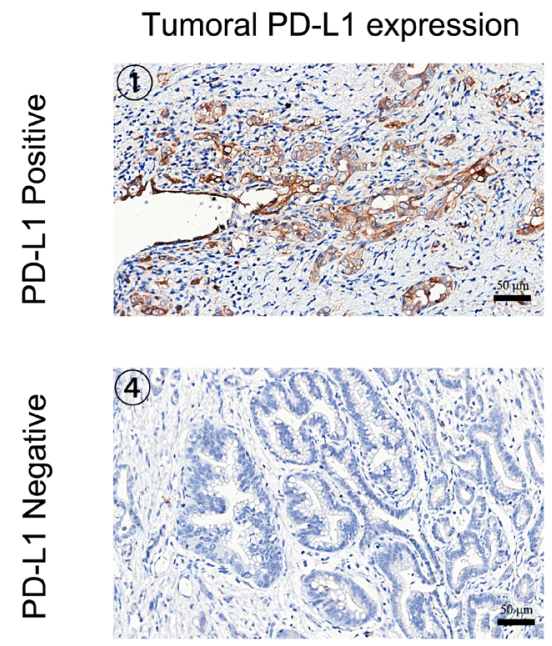

Intra-tumoral CD8+ TIL
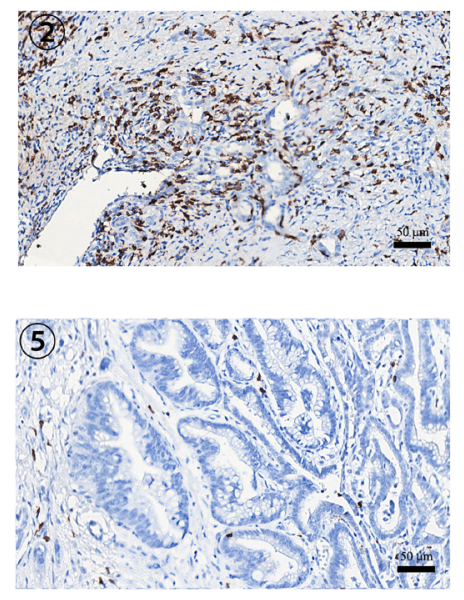

Peri-tumoral CD8+ TIL
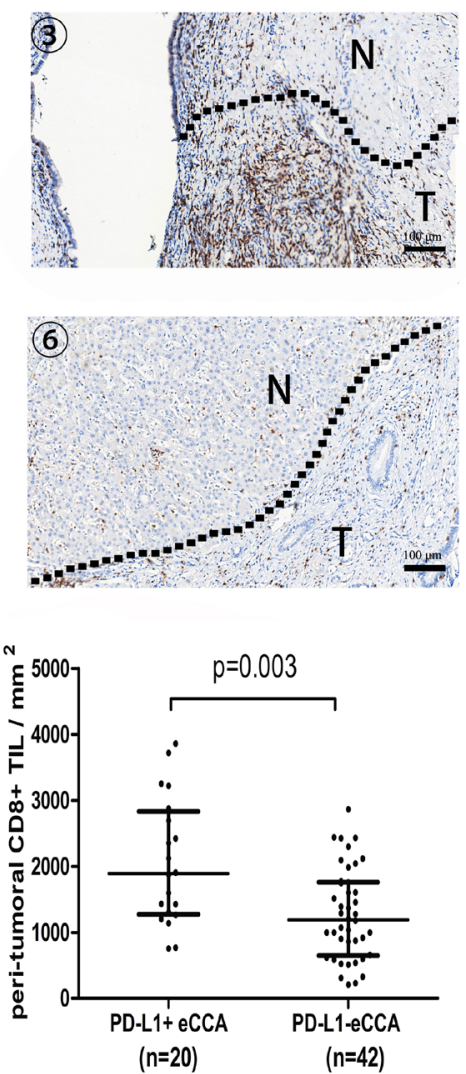

Figure 4. Expression of PD-L1 correlated with TIL density (CD8+). Representative immunohistochemical images of PD-L1 and CD8 show that peri/intra - tumoral CD8+ TIL density was higher in tumors with positive expression of PD-L1 than in the tumors with negative expression of PD-L1 (A). Figures were taken from the same areas in serial sections (A(1) and A(2), A(4) and A(5), respectively. The area marked ' $\mathrm{T}$ ' represents eCCA area, and the area marked ' $\mathrm{N}$ ' represents normal extrahepatic bile duct (A 3) or normal hepatic tissue (A(6). Mann-Whitney $U$-test reveals that intra-tumoral CD3+ and peri/intra - tumoral CD8+ TIL density was higher in eCCA cases showing positive expression of PD-L1 (B).

subpopulations with CD3, CD4 or CD8 antibodies was performed separately. In 62 eCCA cases, patients with positive expression of PD-L1 had higher density of intra-tumor CD3+ and CD8+ TIL (Mann-Whitney $U$-test; $P=0.002$ and $P<0.001$ respectively), and higher peri-tumor CD8+ TIL (Mann-Whitney $U$-test; $P$ $=0.003$ ) (Figure 4B). However, there was no significant difference of CD4+ TIL density between positive and negative PD-L1 expressions. When serial sections from eCCA were stained with the anti-PD-L1 antibodies, tumoral PD-L1 staining and accumulated stromal CD8+ immune cells were present in the same region of each tissue. (Figure 4A).

As PD-L1 attenuates antitumor immune system depending on PD-1/PD-L1 protein-protein interaction, we performed double immunofluorescence staining for PD-L1/PD-1 for the sample with PD-L1 positive expression $(n=20)$. In all PD-L1 positive tumors, a positive membranous PD-1 expression was found on cells in the PD-L1 positive tumor site (Figure 5A). The relative proximity of $\mathrm{PD}-1$ to $\mathrm{PD}-\mathrm{L} 1$ provides evidence of a direct contact between PD-1-positive and PDL1-positive cells. It is known that in vitro, PD-L1 can be strongly induced on the surface of cancer cells by adding IFN- $\gamma$ (28). Using double immunofluorescence staining, we observed that IFN- $\gamma$ was concentrated between PD-L1 positive tumors and the TILs (Figure 5B). PD-L1 expression on cancer cells in eCCA may therefore be caused by IFN- $\gamma$

\section{3. eCCA exhibits different patterns of HLA Class I expression in the tumor microenvironment}

As HLA class I molecules are involved in cytotoxic T cell recognition of malignant cells, we determined its expression profile in eCCA by immunohistochemistry, using antibodies against classical HLA class I molecules (monoclonal anti-pan HLA class I antibody, EMR8-5, which recognizes HLA class I -A, -B, and -C). Staining patterns on normal biliary epithelial cells from extrahepatic bile duct were also evaluated (Figure 6). Interestingly, HLA class I was positive on the cell membrane of normal biliary epithelial cells and the same expression pattern was detected in only 31 $(50 \%)$ of tumor lesions. HLA class I molecule defect was observed in $31(50 \%)$ of lesions, heterogeneous in $13(21.0 \%)$ and negative in 18 (29.0\%). Based on the basal level of PD-L1 expression in normal tissue, 
A

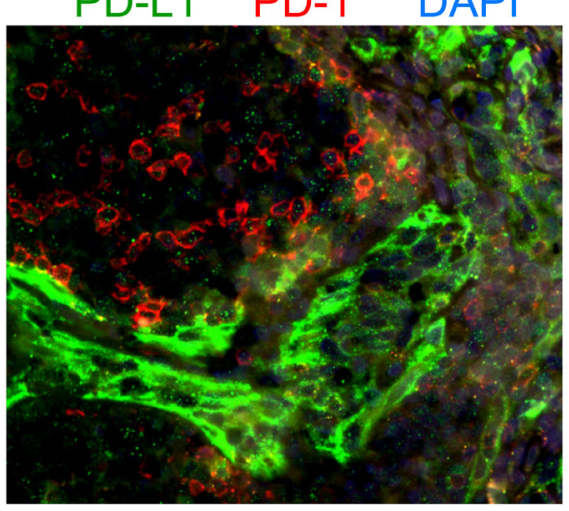

B

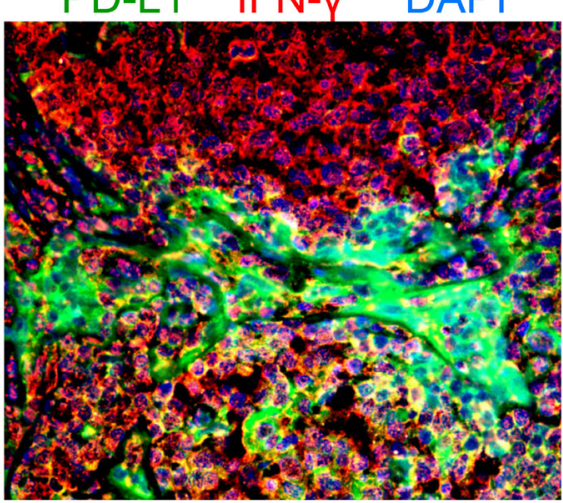

Figure 5. Representative double immunofluorescence staining of PD-L1 (green) and PD-1 (red) (A), and PD-L1 (green) and IFN- $\gamma($ red) (B).
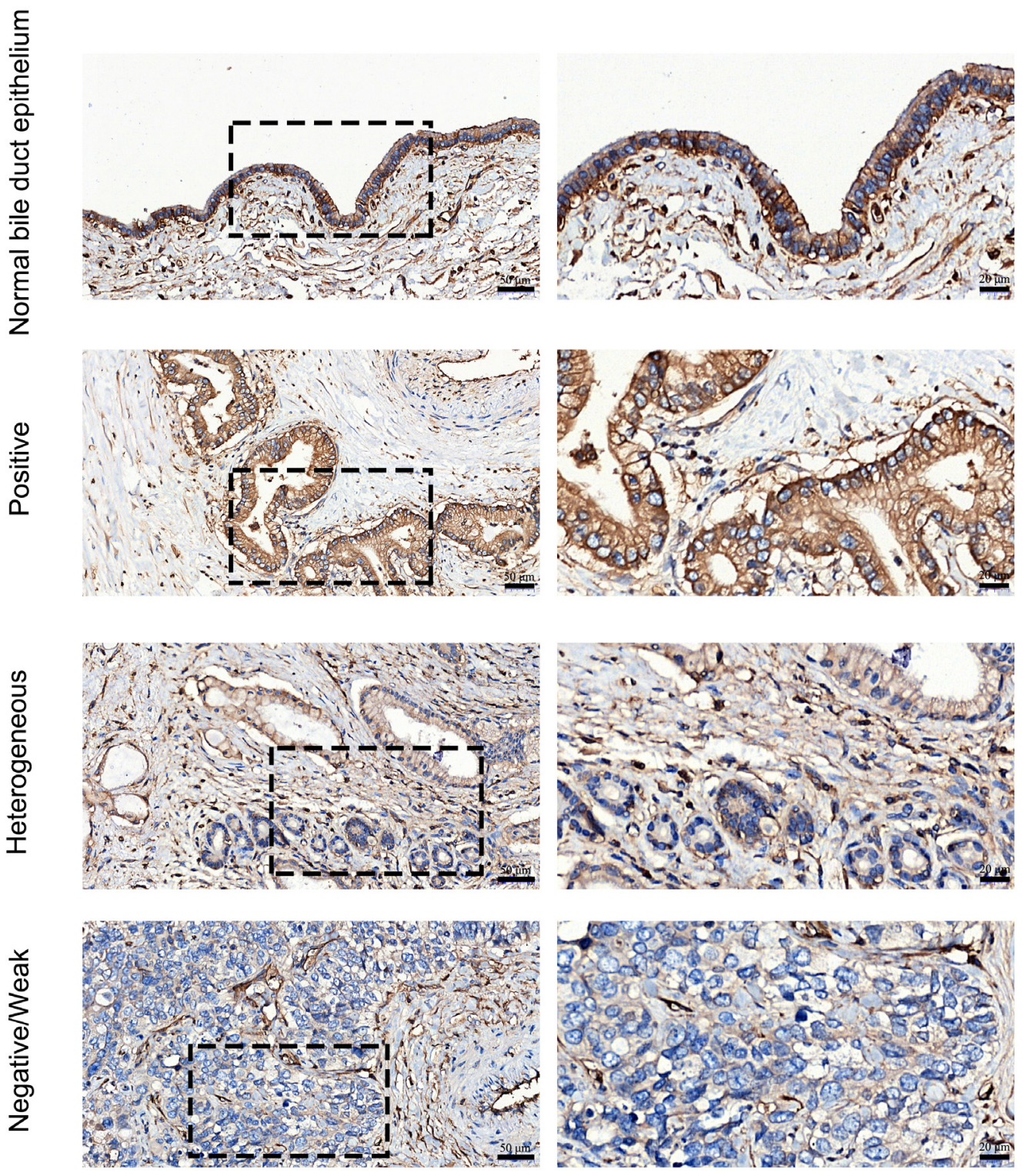

Figure 6. HLA class I expression in eCCA. Representative staining patterns of HLA class I expression in normal biliary epithelial cells and eCCA using immunohistochemistry. In general, basal level of positive HLA class I expression was found on normal biliary epithelial cells. 
A
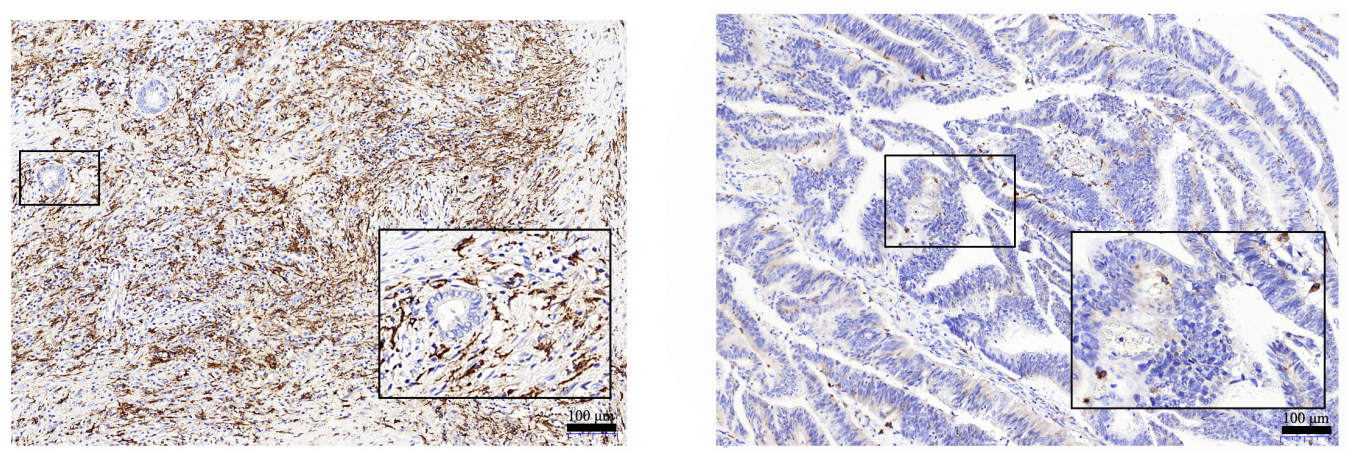

B
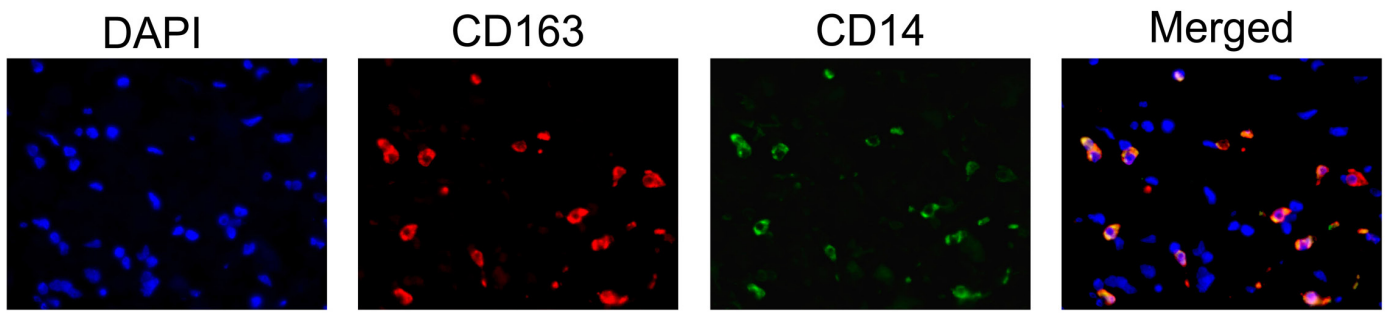

Figure 7. CD163 infiltrate in eCCA. eCCA with low CD163 infiltrate $(<20 \%)$ and high CD163 infiltrate $(>20 \%)(\mathbf{A})$. Characterization of tumor-associated macrophages in eCCA (B). Antibodies against CD14 (green) and CD163 (red) were used to detect macrophages and characterize their polarization as CD14+CD163-represented M1 type, while M2CD14+CD163+ represented M2.

our data demonstrated that HLA class I molecule downregulation is a frequent phenomenon observed in eCCA.

In 62 eCCA cases evaluated, CD8+ TIL density was higher in the high HLA-I expression group (positive and heterogeneous expression, $n=43$ ) than that in the low HLA-I expression group (absolutely negative expression, $n=18$ ) (Mann-Whitney $U$-test; $P=0.028$,) (Figure S3, http://www.biosciencetrends.com/action/ getSupplementalData.php?ID=37). Additionally, high HLA-I expression was significantly associated with upregulated PD-L1 expression of tumor cells $(P$ $<0.001$ ). However, no other associations were found between other clinicopathological parameters and HLA-I expression (Table S1, http://www.biosciencetrends.com/ action/getSupplementalData.php?ID=37).

\section{4. eCCA-infiltrating macrophages mainly exhibit M2- phenotype}

TAM have often been classified into two subtypes: M1 (tumor killing macrophages) and M2 (tumor promoting macrophages) (29). The M2 macrophages in the tumor microenvironment highly associated with tumor-promoting properties and thus evaluation of M2 macrophages in eCCA is important. On 62 tumors evaluated, 46 were highly infiltrated by M2 macrophages in relation to the expression of CD163 $(74.2 \%)$ (Figure 7A). By using a combination of immunofluorescent staining, M2 macrophages represented the predominant macrophage population in eCCA as nearly all CD14 positive cells also expressed CD163, which acted as a M2-like surface profile (Figure 7B). However, no impact of M2 macrophage infiltration on PD-L1 expression or clinicopathological characteristics was observed (Table S2, http://www. biosciencetrends.com/action/getSupplementalData. php? ID=37).

\section{Discussion}

The past decade of cancer research has generated encouraging results for cancer prevention by directly targeting the immune system using immune modulating agents (30). PD-1/PD-L1 checkpoint blockade is of considerable interest, being capable of eliciting durable responses in several different malignancies including, but not limited to, melanoma, lung and renal cancers (31). Whereas PD-L1/PD-1 inhibitor therapy is an active field of clinical research, few studies have attempted to evaluate the feasibility of targeting PD-L1/PD-1 pathway in CCA, because of its rarity (32). Furthermore, immune response in tumor microenvironment is complex, and PD-L1 expression alone may not represent the whole predictive value. Infiltrating lymphocytes and immune-modulatory molecules in tumor sites, such as MHC, have been noticed to associate with treatment effects of anti-PD-1/ PD-L1 therapy (20). Thus, a better understanding of the eCCA immune microenvironment may be essential 
to optimize and to personalize immunotherapies, as well as to precisely predict their efficacies in current immunotherapies.

A limited number of papers have assessed PD-L1 expression in eCCA on particular demographic groups, suggesting an East-West difference in expression pattern of PD-L1. Takashi Ueno et al., using two different monoclonal antibodies against PD-L1, described its expression in Japanese patients being $36 \%$ and $45 \%$, respectively (33). MA et al. found similar PD-L1 expression in Chinese cases (30/70, $42.86 \%$ ) (22). In contrast, the work by Dirk Walter et al., focused on Caucasian cohorts and reported a low frequency of PD-L1 expression $(8 / 69,11.6 \%)$ in their patients (16). Our screening of PD-L1 expression by immunohistochemistry in Chinese eCCA revealed that the rate of PD-L1-positive tumors was $32.3 \%(20 / 62)$, which was consistent with the Asian cohorts, and again found to be higher than the Caucasian group. Considering we used clone anti-PD-L1 antibodies and the method to assess cell surface PD-L1 expression following Dirk Walter's protocol used in Caucasian samples, the discrepancy might be race/ethnicityrelated. Interestingly, an integrated genomic and transcriptomic analysis of 582 Asian and 256 Caucasian primary liver cancers (hepatocellular carcinoma and cholangiocarcinoma) has identified vast race/ethnicityrelated heterogeneity suggesting Asian CCA were more likely to be infiltrated by $\mathrm{T}$ cells (17). Since tumor-T cell interaction is an important prerequisite for tumor PD-L1 expression, this finding may explain in part the higher levels of PD-L1 expression in Asian eCCA compared with Caucasian eCCA.

To date, two regulator mechanisms of PD-L1 induction have been proposed: intrinsic and extrinsic mechanisms (28). Intrinsic induction of PD-L1 is due to oncogenic-driven pathways, demonstrating no correlation with innate immune status (34). In contrast to the intrinsic mechanism, extrinsic induction of PDL1 in tumor site is an adaptation to an on-going antitumor immunity, a process termed adaptive resistance (28). Specifically, the released pro-inflammatory cytokines, particularly IFN- $\gamma$ from activated T cells trigger the expression of PD-L1 on cancer cells (35). PD-L1 binding to PD-1 on T cells that on the one hand leads to $\mathrm{T}$ cell exhaustion can also be targeted by PD-L1/PD-L1 blockades to reactivate an antitumor response (35). Additionally, accumulating evidence suggested that tumor microenvironment characterized by inflammatory infiltration act as a precondition for PD-1/PD-L1 checkpoint blockades (36). In our eCCA study, the PD-L1 positivity was associated with significantly increased intra-tumoral CD3+ and CD8+ TILs infiltrate, indicating an immuneactive microenvironment in $\mathrm{PD}-\mathrm{L} 1$ positive eCCA. Additionally, PD-L1 expression was predominantly restricted to the regions where accumulated stromal
CD8+ TILs are present, reaffirming the colocalization of inflammatory response with PD-L1 expression in eCCA. Expectedly, the current study showed that PD-L1 positive eCCA cells were adjacent to PD-1positive cells and surrounded by IFN- $\gamma$. Taken together, our findings suggest that upregulation of PD-L1 in eCCA is due to an adaptive immune response based on the association of PD-L1 and tumor-infiltrating lymphocytes including CD8-positive TILs and on the finding of colocalization of proinflammatory cytokines. Furthermore, our finding that PD-L1 positive eCCA cells were adjacent to PD-1 positive TILs predicted a greater chance of response to PD-L1/PD-1 checkpoint blockade therapy.

Considering the immune evasion mechanism by PD-1/PD-L1 pathway, PD-L1 overexpression was generally expected to be linked with progression and poor prognosis (37). While others claimed conflicting results, colorectal cancer for example, PD-L1 positive was reported to be associated with low malignant features and a longer disease-free survival or overall survival (38). Similarly, the clinical association of PD-L1 expression remained controversial in eCCA studies, especially survival. MA et al. showed in a series of 70 eCCA patients that PD-L1 expression was associated with advanced TNM stage, lymphatic metastasis and an unfavorable outcome (22). However, Dirk Walter reported no prognosis difference between PD-L1 positive and PD-L1 negative expression in 69 eCCA patients (16). In this study, we assess the prognostic role of PD-L1 expression in 62 patients with eCCA and observed that PD-L1 expression was associated with absence of vascular invasion and better outcome. These inconsistencies may be explained by a restricted number of cases in the current eCCA studies, thereby resulting in an inadequate statistical efficacy. More recently, a large scale comprehensive genomic study involving nearly 500 CCAs showed an improved survival in patients with specific upregulation of immune checkpoint genes (39). Considering the complex dynamics from initiate anti-tumor immune responses to adaptive immune resistance, we prefer the view of PD-L1 as a positive marker for prognosis. In contrast to CTLA-4, which attenuates the initial $\mathrm{T}$ cell activation stage, PD-L1 is induced as a protective response to pre-existing successful anti-tumor immunity (40). On theoretical grounds, despite that PD-L1 positive tumor cells have become protected from cellular immune attack, activated TILs in tumor microenvironment may still have a positive impact on survival by recognition and destruction of other PD-L1 negative cells. Moreover, our study found that the PDL1 expression was significantly associated with absence of vascular invasion, which reinforces the notion that on-going antitumor immunity to prevent the spread of malignant cells remained in PD-L1 positive eCCA, thereby improving the clinical outcome of patients in 
our study.

HLA class I molecules, composed of a polymorphic heavy chain encoded by HLA-A, -B, and -C genes, are expressed by most cell types and present peptides derived from cellular proteins to CD8+ T cells (41). As a prerequisite for recognition of malignant cells, normal expression of HLA class I molecules on tumor cell surface is crucial to evoke anti-tumor immune responses (42). Moreover, the preexisting HLA class I level in the tumor region has a vital role in determining the efficiency of cancer immunotherapy (43). Unfortunately, current evidence indicated that loss or selective downregulation of HLA expression is not random but a frequent finding in various types of cancer, which has been described from $16 \%$ for cutaneous melanoma to $50 \%$ for prostate cancer (44). However, no data of HLA expression in eCCA had been published so far. We report herein for the first time that a significant percentage of eCCA are heterogeneous (21.0\%) or total loss $(29.0 \%)$. Generation of MHC-I deficient tumor cells is believed to be a consequence of continuous exposure to the host's immune response (44). Conversely, established HLA-I expression pattern may also rearrange the anti-tumor response via affecting tumor infiltration, which has been well described in non-small cell lung cancer (25). At early stages, tumors are HLA-I positive/ heterogeneous and are highly infiltrated with lymphocytes; while at later stages, tumor nests are mostly HLA-I negative, which forms a immunosuppressive microenvironment that prevents intra-tumor immune cell infiltration (39). In this regard, it can be theoretically proposed that TILs tend to infiltrate into high HLA-I expression (positive/ heterogeneous) tumors, which undergo longtime interactions with cancer cells, thereby triggering the induction of inhibitory molecule genes. This may explain in our eCCA study why tumor HLA-I expression was strongly correlated with TILs infiltration as well as tumor PD-L1 expression. Interestingly, recent integrative clustering of gene expression in 489 CCA samples has uncovered concordant results that a distinct subtype demonstrating upregulation of immune checkpoint genes also displayed a high level of infiltrating immune cells as well as pathways involved in antigen presentation (39). All together, we deduced that PD-L1 positive eCCA may be susceptible to treatment with immune checkpoint inhibitors for elevated antigen exposure as well as the local proinflammatory state in patients.

Among antigen presenting cells recruited to the tumor site, macrophages are often most abundant although their population is highly heterogeneous (29). Newly recruited macrophages adopt a pro-inflammatory phenotype designated as M1 macrophages, which have roles in mounting anti-cancer responses. However, once the tumor microenvironment is established, the M1 macrophages are educated into a pro-tumor phenotype, M2 macrophages (29). The antitumor mechanisms of M2 macrophages include extracellular matrix remodeling, stimulation of tumor angiogenesis and more importantly inhibition of cytotoxic T-cell responses (45). Indeed, M2 macrophages in tumors are emerging as a key player for the efficacy of PD-1/PDL1 checkpoint blockades and are frequently evaluated in clinical studies (46). In this study, we found that M2 macrophages highly infiltrate into eCCA and are present at either PD-L1 positive tumors or negative tumors. The presence of unusually high numbers of M2 macrophages in eCCA is still uncertain and, further, this recruitment is likely to be more common in hypoxic tumor tissue than normoxic tumor tissues (47). Concerning the dual role of TAMs in tumor sites, therapeutic protocol included the promotion of anticancer functions of M1 macrophages, the reduction of M2 macrophages, and the re-programming of M2 into M1 phenotype (48). Kidney and ovarian cancer are excellent examples of tumors with a distinguishable hybrid phenotype of TAMs that share both M1 and M2 properties, implicating ideal candidates for M1-targeted therapy $(49,50)$. While in eCCA, we found that most macrophages display an M2 phenotype as almost all of the CD14+ cells also co-expressed CD163 protein. Therefore, rather than boosting local protective M1 macrophage responses, ablation or repolarization of M2 macrophages might contribute efficiently to eCCA treatments.

eCCA is a highly malignant carcinoma and so far, the role of chemotherapy or radiotherapy is uncertain (6). In our study, the PD-L1-positive eCCA with characteristics of markedly increased TILs density, notably CD8+ TILs, together with high HLA class I expression represented a group of patients who would benefit from PD-1/PD-L1-targeted therapies. For the other patients, combinations of therapies were likely required to recover HLA class I expression or to active suppression of inflammatory infiltration (34). Of note, the majority of eCCA in our series preferentially accumulated M2 macrophages, which have been proven to confer PD-1/PD-L1 blockade resistance (46). In view of our study and previous literature, we can speculate that the targeting macrophages in various ways within TME may lead to potent efficacy of PD-1/PD-L1 blockade.

Given that eCCA remains a rare disease in the world, the major limitation of our study is the relatively small number of cases and we attempted to assess different available correlations between immune features, clinicopathologic data and outcome. Other limitations include the retrospective design of this study and the lack of data available for response to PD-1/ PD-L1 inhibitors. Future prospective studies with large cohorts of Chinese eCCA patients treated with PD-1/ PD-L1 blockades are required to determine whether our findings are clinically meaningful. 


\section{Acknowledgements}

This study was supported by Beijing Municipal Administration of Hospital's Mission Plan (Code: SML20152201) and Beijing Municipal Administration of Hospital's Clinical Medicine Development of Special Funding Support (Code: ZYLX201712)

\section{References}

1. Razumilava N, Gores GJ. Cholangiocarcinoma. Lancet. 2014; 383:2168-2179.

2. Khan SA, Toledano MB, Taylor-Robinson SD. Epidemiology, risk factors, and pathogenesis of cholangiocarcinoma. HPB (Oxford). 2008; 10:77-82.

3. Khan SA, Emadossadaty S, Ladep NG, Thomas HC, Elliott P, Taylor-Robinson SD, Toledano MB. Rising trends in cholangiocarcinoma: Is the ICD classification system misleading us? J Hepatol. 2012; 56:848-854.

4. DeOliveira ML, Cunningham SC, Cameron JL, Kamangar F, Winter JM, Lillemoe KD, Choti MA, Yeo CJ, Schulick RD. Cholangiocarcinoma: Thirty-one-year experience with 564 patients at a single institution. Ann Surg. 2007; 245:755-762.

5. Esnaola NF, Meyer JE, Karachristos A, Maranki JL, Camp ER, Denlinger CS. Evaluation and management of intrahepatic and extrahepatic cholangiocarcinoma. Cancer. 2016; 122:1349-1369.

6. Khan SA, Davidson BR, Goldin RD, Heaton N, Karani J, Pereira SP, Rosenberg WM, Tait P, Taylor-Robinson $\mathrm{SD}$, Thillainayagam AV, Thomas HC, Wasan H; British Society of Gastroenterology. Guidelines for the diagnosis and treatment of cholangiocarcinoma: An update. Gut. 2012; 61:1657-1669.

7. Darwish Murad S, Kim WR, Harnois DM, et al. Efficacy of neoadjuvant chemoradiation, followed by liver transplantation, for perihilar cholangiocarcinoma at 12 US centers. Gastroenterology. 2012; 143:88-98.e83; quiz e14.

8. Schweitzer N, Vogel A. Systemic therapy of cholangiocarcinoma: From chemotherapy to targeted therapies. Best Pract Res Clin Gastroenterol. 2015; 29:345-353.

9. Massani M, Nistri C, Ruffolo C, Bonariol R, Pauletti B, Bonariol L, Caratozzolo E, Morana G, Bassi $\mathrm{N}$. Intrahepatic chemotherapy for unresectable cholangiocarcinoma: review of literature and personal experience. Updates Surg. 2015; 67:389-400.

10. Jiao Y, Pawlik TM, Anders RA, et al. Exome sequencing identifies frequent inactivating mutations in BAP1, ARID1A and PBRM1 in intrahepatic cholangiocarcinomas. Nat Genet. 2013; 45:1470-1473.

11. Hay M, Thomas DW, Craighead JL, Economides C, Rosenthal J. Clinical development success rates for investigational drugs. Nat Biotechnol. 2014; 32:40-51.

12. Topalian SL, Drake CG, Pardoll DM. Immune checkpoint blockade: A common denominator approach to cancer therapy. Cancer cell. 2015; 27:450-461.

13. Mahoney KM, Freeman GJ, McDermott DF. The next immune-checkpoint inhibitors: PD-1/PD-L1 blockade in melanoma. Clin Ther. 2015; 37:764-782.

14. Menon S, Shin S, Dy G. Advances in Cancer Immunotherapy in Solid Tumors. Cancers (Basel). 2016;
8. pii: E106.

15. Rizvi S, Khan SA, Hallemeier CL, Kelley RK, Gores GJ. Cholangiocarcinoma - evolving concepts and therapeutic strategies. Nat Rev Clin Oncol. 2018; 15:95-111.

16. Walter D, Herrmann E, Schnitzbauer AA, Zeuzem S, Hansmann ML, Peveling-Oberhag J, Hartmann S. PDL1 expression in extrahepatic cholangiocarcinoma. Histopathology. 2017; 71:383-392.

17. Chaisaingmongkol J, Budhu A, Dang H, et al. Common Molecular Subtypes Among Asian Hepatocellular Carcinoma and Cholangiocarcinoma. Cancer cell. 2017; 32:57-70. e53.

18. Enwere EK, Kornaga EN, Dean M, Koulis TA, Phan T, Kalantarian M, Kobel M, Ghatage P, Magliocco AM, Lees-Miller SP, Doll CM. Expression of PD-L1 and presence of $\mathrm{CD} 8$-positive $\mathrm{T}$ cells in pre-treatment specimens of locally advanced cervical cancer. Mod Pathol. 2017; 30:577-586.

19. Maruse Y, Kawano S, Jinno T, Matsubara R, Goto Y, Kaneko N, Sakamoto T, Hashiguchi Y, Moriyama M, Toyoshima T, Kitamura R, Tanaka H, Oobu K, Kiyoshima T, Nakamura S. Significant association of increased PD-L1 and PD-1 expression with nodal metastasis and a poor prognosis in oral squamous cell carcinoma. Int J Oral Maxillofac Surg. 2018; 47:836845 .

20. Zhao X, Subramanian S. Intrinsic resistance of solid tumors to immune checkpoint blockade therapy. Cancer Res. 2017; 77:817-822.

21. Garrido F, Ruiz-Cabello F, Aptsiauri N. Rejection versus escape: The tumor MHC dilemma. Cancer Immunol Immunother. 2017; 66:259-271.

22. Ma K, Wei X, Dong D, Wu Y, Geng Q, Li E. PD-L1 and $\mathrm{PD}-1$ expression correlate with prognosis in extrahepatic cholangiocarcinoma. Oncol Lett. 2017; 14:250-256.

23. Katsuya Y, Fujita Y, Horinouchi H, Ohe Y, Watanabe $\mathrm{S}$, Tsuta K. Immunohistochemical status of PD-L1 in thymoma and thymic carcinoma. Lung Cancer. 2015; 88:154-159.

24. Zhang QW, Liu L, Gong CY, Shi HS, Zeng YH, Wang XZ, Zhao YW, Wei YQ. Prognostic significance of tumor-associated macrophages in solid tumor: A metaanalysis of the literature. PloS One. 2012; 7:e50946.

25. Kostine M, Briaire-de Bruijn IH, Cleven AHG, Vervat C, Corver WE, Schilham MW, Van Beelen E, van Boven H, Haas RL, Italiano A, Cleton-Jansen AM, Bovée JVMG. Increased infiltration of M2-macrophages, T-cells and PD-L1 expression in high grade leiomyosarcomas supports immunotherapeutic strategies. Oncoimmunology. 2018; 7:e1386828.

26. Garrido F, Perea F, Bernal M, Sanchez-Palencia A, Aptsiauri N, Ruiz-Cabello F. The Escape of Cancer from T Cell-Mediated Immune Surveillance: HLA Class I Loss and Tumor Tissue Architecture. Vaccines (Basel). 2017; 5. pii: E7.

27. Quero L, Hanser E, Manigold T, Tiaden AN, Kyburz D. TLR2 stimulation impairs anti-inflammatory activity of M2-like macrophages, generating a chimeric M1/M2 phenotype. Arthritis Res Ther. 2017; 19:245.

28. Sanmamed MF, Chen L. Inducible expression of B7H1 (PD-L1) and its selective role in tumor site immune modulation. Cancer J. 2014; 20:256-261.

29. Lee HW, Choi HJ, Ha SJ, Lee KT, Kwon YG. Recruitment of monocytes/macrophages in different tumor microenvironments. Biochim Biophys Acta. 2013; 
1835:170-179.

30. Kyi C, Postow MA. Checkpoint blocking antibodies in cancer immunotherapy. FEBS Lett. 2014; 588:368-376.

31. Drake CG, Lipson EJ, Brahmer JR. Breathing new life into immunotherapy: Review of melanoma, lung and kidney cancer. Nat Rev Clin Oncol. 2014; 11:24-37.

32. Abou-Alfa GK, Andersen JB, Chapman W, et al. Advances in cholangiocarcinoma research: report from the third Cholangiocarcinoma Foundation Annual Conference. J Gastrointest Oncol. 2016; 7:819-827.

33. Ueno T, Tsuchikawa T, Hatanaka KC, Hatanaka Y, Mitsuhashi T, Nakanishi Y, Noji T, Nakamura T, Okamura K, Matsuno Y, Hirano S. Prognostic impact of programmed cell death ligand 1 (PD-L1) expression and its association with epithelial-mesenchymal transition in extrahepatic cholangiocarcinoma. Oncotarget. 2018; 9:20034-20047.

34. Zhang Y, Chen L. Classification of advanced human cancers based on tumor immunity in the MicroEnvironment (TIME) for cancer immunotherapy. JAMA Oncol. 2016; 2:1403-1404.

35. Zou W, Chen L. Inhibitory B7-family molecules in the tumour microenvironment. Nat Rev Immunol. 2008; 8:467-477.

36. Day D, Monjazeb AM, Sharon E, Ivy SP, Rubin EH, Rosner GL, Butler MO. From famine to feast: Developing early-phase combination immunotherapy trials wisely. Clin Cancer Res. 2017; 23:4980-4991.

37. Wu P, Wu D, Li L, Chai Y, Huang J. PD-L1 and survival in Ssolid tumors: A meta-analysis. PloS One. 2015; 10:e131403.

38. Wei XL, Wu QN, Chen DL, Zeng ZL, Lu JB, Liu ZX, Ju HQ, Ren C, Pan ZZ, Wang FH, Xu RH. The clinical and biomarker association of programmed death ligand 1 and its spatial heterogeneous expression in colorectal cancer. J Cancer. 2018; 9:4325-4333.

39. Jusakul A, Cutcutache I, Yong CH, et al. Whole-Genome and Epigenomic Landscapes of Etiologically Distinct Subtypes of Cholangiocarcinoma. Cancer Discov. 2017; 7:1116-1135.

40. Parry RV, Chemnitz JM, Frauwirth KA, Lanfranco AR, Braunstein I, Kobayashi SV, Linsley PS, Thompson CB, Riley JL. CTLA-4 and PD-1 receptors inhibit T-cell activation by distinct mechanisms. Mol Cell Biol. 2005; 25:9543-9553.

41. Tang Q, Nie F, Kang J, Ding H, Zhou P, Huang J. NIEluter: Predicting peptides eluted from HLA class I molecules. J Immunol Methods. 2015; 422:22-27.

42. del Campo AB, Carretero J, Aptsiauri N, Garrido F. Targeting HLA class I expression to increase tumor immunogenicity. Tissue Antigens. 2012; 79:147-154.

43. Aptsiauri N, Carretero R, Garcia-Lora A, Real LM, Cabrera T, Garrido F. Regressing and progressing metastatic lesions: resistance to immunotherapy is predetermined by irreversible HLA class I antigen alterations. Cancer Immunol Immunother. 2008; 57:17271733.

44. Chang CC, Campoli M, Ferrone S. HLA class I defects in malignant lesions: what have we learned? Keio J Med. 2003; 52:220-229.

45. Qian BZ, Pollard JW. Macrophage diversity enhances tumor progression and metastasis. Cell. 2010; 141:39-51.

46. Santoni M, Romagnoli E, Saladino T, Foghini L, Guarino S, Capponi M, Giannini M, Cognigni PD, Ferrara G, Battelli N. Triple negative breast cancer: Key role of Tumor-Associated Macrophages in regulating the activity of anti-PD-1/PD-L1 agents. Biochim Biophys Acta Rev Cancer. 2018; 1869:78-84.

47. Raggi F, Pelassa S, Pierobon D, Penco F, Gattorno M, Novelli F, Eva A, Varesio L, Giovarelli M, Bosco MC. Regulation of Human Macrophage M1-M2 Polarization Balance by Hypoxia and the Triggering Receptor Expressed on Myeloid Cells-1. Front Immunol. 2017; 8:1097.

48. Noy R, Pollard JW. Tumor-associated macrophages: from mechanisms to therapy. Immunity. 2014; 41:49-61.

49. Kovaleva OV, Samoilova DV, Shitova MS, Gratchev A. Tumor Associated Macrophages in Kidney Cancer. Anal Cell Pathol (Amst). 2016; 2016:9307549.

50. Zhang M, He Y, Sun X, Li Q, Wang W, Zhao A, Di W. A high M1/M2 ratio of tumor-associated macrophages is associated with extended survival in ovarian cancer patients. J Ovarian Res. 2014; 7:19.

(Received January 11, 2019; Revised January 29, 2019; Accepted February 9, 2019) 